\title{
MicroRNA expression profiles predict progression and clinical outcome in lung adenocarcinoma
}

This article was published in the following Dove Press journal:

OncoTargets and Therapy

15 September 2016

Number of times this article has been viewed

\author{
Kang Lin ${ }^{1, *}$ \\ Tao Xul,* \\ Bang-Shun $\mathrm{He}^{\prime}$ \\ Yu-Qin Pan' \\ Hui-Ling Sun' \\ Hong-Xin Peng ${ }^{2}$ \\ Xiu-Xiu $\mathrm{Hu}^{2}$ \\ Shu-Kui Wang' \\ 'Central Laboratory, Nanjing First \\ Hospital, Nanjing Medical University, \\ ${ }^{2}$ Medical School, Southeast University, \\ Nanjing, Jiangsu, People's Republic \\ of China \\ *These authors contributed equally \\ to this work
}

Correspondence: Shu-Kui Wang Central Laboratory, Nanjing First Hospital, Nanjing Medical University, 68 Changle Road, 210006 Nanjing, Jiangsu, People's Republic of China Tel/fax +86 2552887003

Email shukwang@।63.com

\begin{abstract}
Lung cancer is one of the leading causes of cancer death worldwide. Accumulating evidence has indicated that microRNAs (miRNAs) can be proposed as promising diagnostic and prognostic markers for various cancers. The current study analyzed the miRNA expression profiles of 418 lung adenocarcinoma (LUAD) cases obtained from The Cancer Genome Atlas dataset, with the aim to investigate the relationship of miRNAs with progression and prognosis of LUAD. A total of 185 miRNAs were found to be differentially expressed between LUAD tumor tissues and adjacent normal tissues. Among them, 13, 10, 0, and 10 miRNAs were discovered to be associated with pathologic $\mathrm{T}, \mathrm{N}, \mathrm{M}$, and Stage, respectively. Interestingly, mir-200 family (mir-200a, mir-200b, and mir-429) was shown to play a critical role in the progression of LUAD. In the multivariate Cox regression analysis, mir-1468 ( $P=0.009)$, mir-212 $(P=0.026)$, mir-3653 ( $P=0.012)$, and mir-31 ( $P=0.002)$ were significantly correlated with recurrence-free survival. With regard to overall survival, mir-551b $(P=0.011)$, mir-3653 $(P=0.016)$, and mir-31 $(P=0.001)$ were proven as independent prognostic markers. In summary, this study identified the cancer-specific miRNAs that may predict the progression and prognosis of LUAD.
\end{abstract}

Keywords: microRNA, progression, prognosis, lung adenocarcinoma

\section{Introduction}

Lung cancer is one of the most frequently diagnosed cancers and the leading cause of cancer death in both developed and developing countries. ${ }^{1}$ Approximately 733,300 estimated new cases were diagnosed, and 610,200 patients died of lung cancer in 2015 in the People's Republic of China. ${ }^{2}$ In contrast to the steady increase in survival for most cancers, the 5-year relative survival of lung cancer is just $18 \%{ }^{3}$ Among the various histological subtypes, lung adenocarcinoma (LUAD) is the most common type in females and nonsmoking males. ${ }^{1}$ Despite many achievements made in LUAD treatment in the past decade, such as effective molecular targeted therapy, the prognosis of LUAD patients had no significant improvement. ${ }^{4,5}$ Thus, novel cancer-specific biomarkers are urgently needed for LUAD patients, which may help monitor the tumor progression and guide the clinical therapy according to the prediction of survival.

MicroRNAs (miRNAs) are small noncoding RNAs of 18-25 nucleotides, which can bind to the $3^{\prime}$-untranslated region leading to inhibition of translation or degradation of messenger RNAs (mRNAs) at the posttranslational level. ${ }^{6}$ Depending on the targeted mRNAs, miRNAs can act as either tumor oncogenes or tumor suppressors in caners. The dysregulation of miRNAs in lung cancer may lead to alterations in cellular differentiation, proliferation, and apoptosis. ${ }^{7,8}$ Accumulating evidence has demonstrated that miRNAs play a critical role in either progression or prognosis of 
LUAD. ${ }^{9-12}$ However, the majority of previous studies were based on small sample size, relatively limited numbers of miRNAs, or various miRNA detection methods. ${ }^{9,13-16}$

In this study, the authors identified differentially expressed miRNAs between LUAD and adjacent normal tissues with miRNA expression profiles from The Cancer Genome Atlas (TCGA) project. The genome-wide miRNA expression profiles may help identify key miRNAs involved in LUAD carcinogenesis. Furthermore, the authors comprehensively investigated the association between miRNAs and progression and prognosis of LUAD, with the aim to find cancerspecific miRNAs, which may lay the foundation of molecular mechanisms and predict the prognosis of LUAD.

\section{Materials and methods Study population}

From the TCGA data portal (https:/tcga-data.nci.nih.gov/ tcga/tcgaHome2.jsp), ${ }^{17} 522$ LUAD patients pathologically diagnosed from 1991 to 2013 were identified. The corresponding clinical information was downloaded (up to February 28, 2016) and filtered with the following exclusion criteria for further assessment: 1) subjects only with clinical data rather than miRNA expression data and 2) subjects with a history of other malignancies and/or neoadjuvant therapy. A total of 418 LUAD patients were enrolled in this study with complete clinical data, including age, sex, race, smoking status, the American Joint Committee on Cancer (AJCC) TNM staging system, vital status, and follow-up time. A total of 32 participants with adjacent normal tissues were included into Cohort $\mathrm{N}$, while the whole 418 subjects were defined as Cohort T. As the data were downloaded from the TCGA project, all LUAD patients were well informed, and the study was performed in line with the TCGA publication guidelines (http://cancergenome.nih.gov/publications/ publicationguidelines).

\section{Processing miRNA expression profiles}

The miRNA expression data (level 3) of 418 tumor tissues and 32 adjacent normal tissues were downloaded from the TCGA project (up to February 28, 2016). Among the 450 tissues, 400 cases and 50 cases were profiled using the Illumina HiSeq platform and the Illumina Genome Analyzer platform (Illumina Inc., San Diego, CA, USA), respectively. The expression levels of 1,046 miRNAs were presented as reads per million counts and processed with BRB-ArrayTools (Version 4.4.0; National Cancer Institute, Bethesda, MD, USA), which was developed by Doctor Richard Simon and BRB-ArrayTools Development Team. ${ }^{18}$ Before further analysis, brief data filtering was performed according to the following criteria: 1) each miRNA with missing data $>10 \%$ of all samples and 2) each miRNA with $<1.5$ fold change from the median expression value in at least $20 \%$ of total samples was excluded. Finally, the expression levels of retained miRNAs were $\log 2$ transformed.

\section{Statistical analysis}

Clinical variables were measured with chi-square test or Fisher's exact test. The miRNA expression levels between the two different groups (tumor and adjacent normal tissues, $\mathrm{T} 3+\mathrm{T} 4$ and $\mathrm{T} 1+\mathrm{T} 2, \mathrm{~N} 1+\mathrm{N} 2+\mathrm{N} 3$ and N0, M1 and M0, stage III + IV and stage I + II) were evaluated with two-sample $t$-test, while the significance level was set as $P=0.01$ as default to control the false discovery rate. The leave-one-out cross-validation method was utilized to compute misclassification rate of differently expressed miRNAs between tumor and adjacent normal tissues. Unsupervised hierarchical cluster analysis was performed with one minus the centered Pearson correlation and average linkage methods.

Kaplan-Meier method and univariate Cox proportional hazards regression analysis were used to find out the potential prognostic factors for recurrence-free survival (RFS) and overall survival (OS). The median value was used as the cutoff point of miRNA expression levels. Days from initial treatment to new tumor event were defined as RFS, while the total follow-up time was OS. Meanwhile, the permutation test method with 10,000 permutations was used to ensure the robust relationship between miRNAs and survival. Subsequently, the multivariate Cox proportional hazards regression analysis was carried out to identify the independent prognostic markers. A two-sided $P$-value of $<0.05$ was regarded as significant. Statistic analysis was conducted with SPSS 20.0 (IBM Corporation, Armonk, NY, USA) and GraphPad Prism 5.0 (GraphPad Software, Inc., La Jolla, CA, USA).

\section{Results}

\section{Characteristics of patients}

The complete clinical and pathologic variables of all 418 patients are shown in Table 1. The mean age of all these patients was 64.6 years, and the median follow-up time was 23.5 months (inter quartile range, IQR: 14.3-39.9 months). Summarily, 149 patients $(35.6 \%)$ suffered the recurrence after a median follow-up time of 14.6 months (IQR: 8.3-14.6 months), and 150 patients (35.9\%) died after a median follow-up time of 23.2 months (IQR: 11.4-39.9 months). In addition, no significant difference was presented in the distribution of age, sex, race, AJCC TNM staging system, 
Table I Clinical characteristics of patients with lung adenocarcinoma

\begin{tabular}{|c|c|c|c|}
\hline Categories & $\begin{array}{l}\text { Cohort N } \\
(n=32)\end{array}$ & $\begin{array}{l}\text { Cohort T } \\
(n=4 \mid 8)\end{array}$ & $P$-value \\
\hline Age (years), mean $\pm S D$ & $65.0 \pm 10.2$ & $64.6 \pm 10.0$ & 0.823 \\
\hline Sex, n (\%) & & & 0.714 \\
\hline Male & $16(50.0)$ & 195 (46.7) & \\
\hline Female & $16(50.0)$ & $223(53.3)$ & \\
\hline Race, n (\%) & & & 0.099 \\
\hline Asian & $0(0.0)$ & $6(1.4)$ & \\
\hline White & $27(84.4)$ & $3 \mid 4(75.1)$ & \\
\hline African & $5(15.6)$ & $46(1 I .0)$ & \\
\hline NA & $0(0.0)$ & $52(12.5)$ & \\
\hline Pathologic Stage, n (\%) & & & $0.8 \mathrm{II}$ \\
\hline Stage I & $20(62.5)$ & $226(54.1)$ & \\
\hline Stage II & $7(2 \mid .8)$ & $93(22.2)$ & \\
\hline Stage III & $5(15.6)$ & $70(16.7)$ & \\
\hline Stage IV & $0(0.0)$ & $22(5.3)$ & \\
\hline NA & $0(0.0)$ & $7(1.7)$ & \\
\hline Pathologic T, n (\%) & & & 0.900 \\
\hline TI & $13(40.6)$ & 137 (32.8) & \\
\hline $\mathrm{T} 2$ & $16(50.0)$ & $223(53.3)$ & \\
\hline $\mathrm{T} 3$ & $2(6.3)$ & $39(9.3)$ & \\
\hline $\mathrm{T} 4$ & I (3.I) & $16(3.8)$ & \\
\hline $\mathrm{TX}$ & $0(0.0)$ & $3(0.8)$ & \\
\hline Pathologic N, n (\%) & & & 0.520 \\
\hline No & $21(65.6)$ & $269(64.4)$ & \\
\hline $\mathrm{NI}$ & $4(12.5)$ & 75 (I7.9) & \\
\hline N2 & $5(15.6)$ & $62(14.8)$ & \\
\hline N3 & $0(0.0)$ & $2(0.5)$ & \\
\hline NX & $2(6.3)$ & $9(2.2)$ & \\
\hline NA & $0(0.0)$ & $\mathrm{I}(0.2)$ & \\
\hline Pathologic M, n (\%) & & & 0.152 \\
\hline Mo & I8 (56.3) & $284(68.0)$ & \\
\hline MI & $0(0.0)$ & $21(5.0)$ & \\
\hline$M X$ & $14(43.8)$ & $110(26.3)$ & \\
\hline NA & $0(0.0)$ & $3(0.7)$ & \\
\hline Smoking status, n (\%) & & & 0.113 \\
\hline Nonsmoker & $3(9.7)$ & $67(16.0)$ & \\
\hline Reformed smoker & $14(43.8)$ & $235(56.2)$ & \\
\hline Current smoker & $13(40.6)$ & $104(24.9)$ & \\
\hline NA & $2(5.9)$ & $12(2.9)$ & \\
\hline Vital status, n (\%) & & & 0.376 \\
\hline Alive & 23 (7I.9) & $268(64.1)$ & \\
\hline Dead & $9(28.1)$ & $150(35.9)$ & \\
\hline
\end{tabular}

Notes: MX, metastasis status unknown; n, number of patients; NX, regional lymph node unknown; TX, tumor invasion unknown.

Abbreviations: NA, not available; SD, standard deviation.

smoking status, and vital status between Cohort $\mathrm{T}$ and Cohort N $(P>0.05)$.

\section{Differentially expressed miRNAs between LUAD and adjacent normal tissues}

After analysis of 1,046 miRNAs expression in LUAD tumor tissues (Cohort $\mathrm{T}$ ) and adjacent normal tissues (Cohort $\mathrm{N}$ ), a total of 185 miRNAs were found to be differentially expressed by class comparison (Table S1). Among these 185 miRNAs, 133 miRNAs (71.9\%) were upregulated, and the remaining 52 miRNAs (28.1\%) were downregulated. In relation to the fold change, 48 differentially expressed miRNAs showed more than threefold change or $<0.33$-fold change. Among these 48 miRNAs, 33 miRNAs (68.8\%) were upregulated, while 15 miRNAs (31.2\%) were downregulated (Figure 1A). Meanwhile, to examine the reliability of class comparison, class prediction was conducted among all subjects with these 185 miRNAs. A total of 97.1\% (437/450) of these subjects were classified correctly into the unpaired condition. Furthermore, the unsupervised hierarchical clustering was conducted with the 185 miRNAs to separate the tumor and adjacent normal tissues (Figure 1B).

\section{MiRNAs related to progression of LUAD}

Among these 185 differentially expressed miRNAs, class comparison analysis was performed to determine miRNAs associated with AJCC TNM staging system. Overall, 13, 10, 0 , and 10 miRNAs were discovered to be associated with pathologic T, N, M, and Stage, respectively (Tables S2-S4). As described in a Venn diagram (Figure 2), mir-429 was the only one that significantly associated with three variables (pathologic T, pathologic N, and pathologic Stage). Additionally, two miRNAs (mir-30e and mir-3200) were correlated with pathologic $T$ and pathologic Stage, while four miRNAs (mir-600, mir-200a, mir-200b, and mir-548b) were linked to pathologic $\mathrm{N}$ and pathologic Stage.

\section{MiRNAs with prognostic value in LUAD}

To identify miRNAs with prognostic value in LUAD, the 185 differentially expressed miRNAs were subjected to univariate Cox proportional hazard regression analysis. Five miRNAs (mir-548v, mir-1468, mir-212, mir-3653, and mir-31) were significantly associated with RFS (Table 2), while eight miRNAs (mir-548v, mir-30e, mir-1468, mir551b, mir-212, mir-3607, mir-3653, and mir-31) were significantly related to OS (Table 3). Furthermore, KaplanMeier survival analysis and log-rank test were conducted (Figures 3 and 4). After adjusted by multivariate Cox proportional hazards regression analysis, pathologic $\mathrm{T}$ $(P=0.001), \mathrm{N}(P=0.003)$, mir-1468 $(P=0.009)$, mir-212 $(P=0.026)$, mir-3653 $(P=0.012)$, and mir-31 $(P=0.002)$ were found to be independent prognostic factors for RFS (Table 2$)$. With regard to OS, pathologic T $(P=0.002)$, Stage $(P=0.008)$, mir-551b $(P=0.011), \operatorname{mir}-3653(P=0.016)$, and mir-31 $(P=0.001)$ were proven as independent prognostic markers (Table 3). 
A

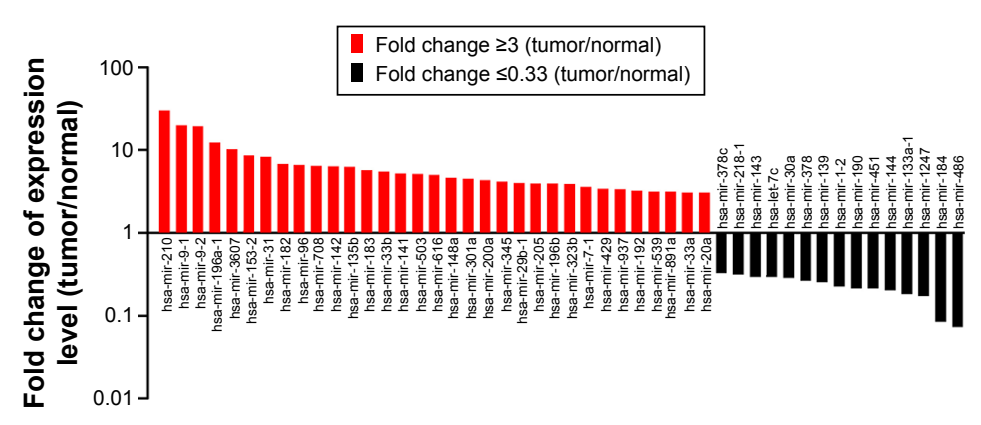

B

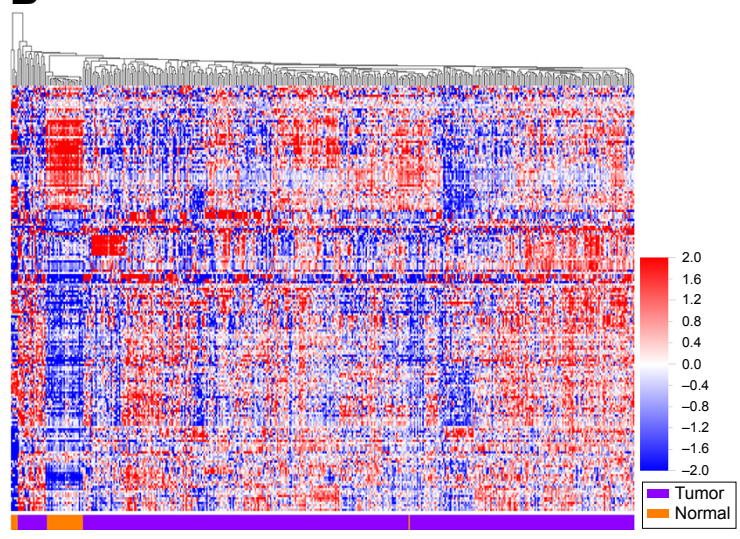

Figure I Differentially expressed microRNAs between lung adenocarcinoma and adjacent normal tissues.

Notes: (A) Differentially expressed microRNAs with more than threefold change or $<0.33$-fold change. (B) Unsupervised hierarchical cluster analysis of the I85 microRNAs expression data.

\section{Discussion}

miRNAs show aberrant expression profiles in various cancers, and dysregulation of miRNAs may alter the neoplastic progression and prognosis of human malignancies. ${ }^{19,20}$ As the previous studies described, miRNA expression data were open access in the TCGA data portal, and analyzing the TCGA data has already become an important approach to investigate the association between miRNA expression patterns and pathologic characteristics and survival in

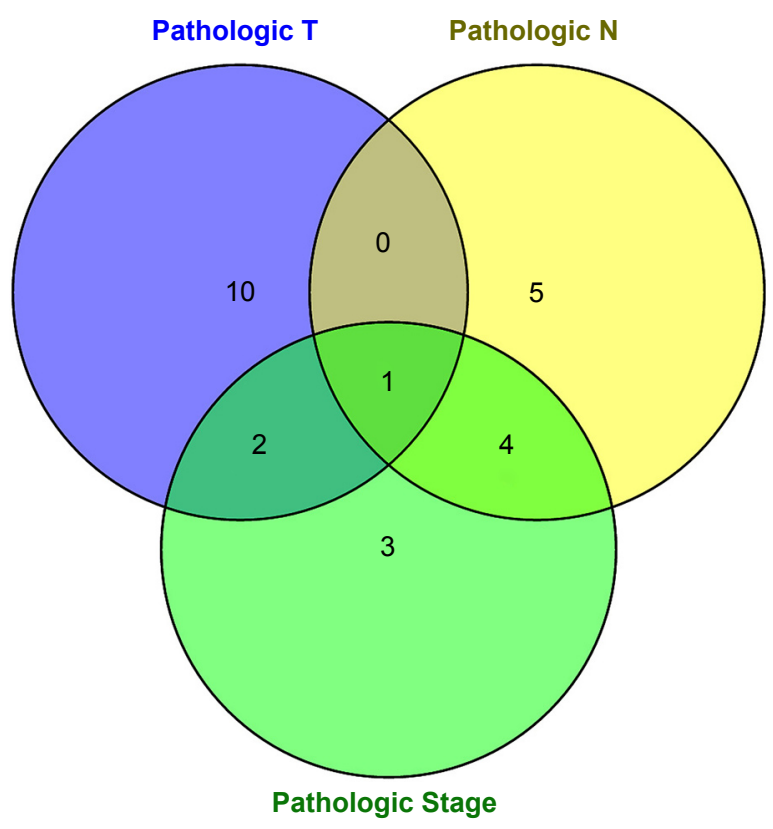

Figure 2 Venn diagram of microRNAs related to progression of lung adenocarcinoma.

Notes: A total of 13, 10, and 10 microRNAs were discovered to be associated with pathologic T, N, and Stage among the 185 differentially expressed miRNAs, respectively.

Abbreviations: $\mathrm{N}$, regional lymph node invasion; $\mathrm{T}$, tumor invasion. various cancer types, including lung cancer, ${ }^{12}$ hepatocellular carcinoma, ${ }^{21}$ renal cell carcinoma, ${ }^{22}$ and bladder cancer. ${ }^{23}$ For LUAD patients, Li et al ${ }^{12}$ identified an eight-miRNA signature for predicting the OS by mining the TCGA data before. However, subjects with a history of other malignancies and/or neoadjuvant therapy were enrolled into that study, which may alter the miRNA expression levels in these LUAD subjects compared to others. In terms of the TCGA LUAD cohort, patients with a history of other malignancies and/or neoadjuvant therapy approximately accounted for $20 \%$, which should not be ignored. Furthermore, cases with OS $<1$ month were removed, which may affect the results of survival analysis. Ge et $\mathrm{al}^{22}$ divided cases into two groups (follow-up time $>30$ days and $<30$ days) in the previous study and found that the survival-related miRNAs differed according to whether these cases with OS $<1$ month were included. Thus, given the differences in the approaches for the selection of cases and miRNAs, the authors identified differentially expressed miRNAs between LUAD and adjacent normal tissues with the TCGA dataset following the strict exclusion criteria and explored the cancer-specific miRNAs for predicting the development and survival in patients with LUAD.

In the present study, 185 miRNAs were identified to be differentially expressed between LUAD tumor tissues and adjacent normal tissues, which clearly separated the tumor and adjacent normal tissues in the unpaired condition. Subsequently, 13, 10, and 10 miRNAs were, respectively, discovered to be associated with pathologic T, N, and Stage among these 185 differentially expressed miRNAs. Unfortunately, none was significantly related to tumor metastasis status, which may be explained by the small number of M1 patients accounting for only $5 \%$ in this study. Interestingly, mir-200 
Table 2 Univariate and multivariate Cox regression analyses of recurrence-free survival in lung adenocarcinoma

\begin{tabular}{|c|c|c|c|c|}
\hline \multirow[t]{2}{*}{ Variables } & \multicolumn{2}{|l|}{ Univariate analysis } & \multicolumn{2}{|c|}{ Multivariate analysis } \\
\hline & HR (95\% Cl) & $P$-value ${ }^{a}$ & HR (95\% Cl) & $P$-value ${ }^{a}$ \\
\hline Age ( $\geq 65$ years vs $<65$ years) & $1.002(0.720-1.392)$ & 0.992 & & \\
\hline Sex (male vs female) & $0.961(0.692-1.333)$ & 0.810 & & \\
\hline $\mathrm{T}(\mathrm{T} 3$ + T4 vs TI + T2) & $2.336(1.533-3.560)$ & 0.000 & $2.312(1.380-3.874)$ & 0.001 \\
\hline$N(N 1+N 2$ + N3 vs N0) & $2.403(1.728-3.343)$ & 0.000 & $1.882(1.237-2.866)$ & 0.003 \\
\hline M (MI vs M0) & $2.426(1.347-4.369)$ & 0.003 & & \\
\hline Stage (III + IV vs I + II) & $2.409(1.710-3.394)$ & 0.000 & & \\
\hline hsa-mir-548v (high vs low) & $0.601(0.424-0.852)$ & 0.004 & & \\
\hline hsa-mir-1468 (high vs low) & $0.661(0.473-0.923)$ & 0.015 & $0.550(0.35 I-0.86 I)$ & 0.009 \\
\hline hsa-mir-212 (high vs low) & $1.520(1.094-2.112)$ & 0.013 & $1.624(1.059-2.490)$ & 0.026 \\
\hline hsa-mir-3653 (high vs low) & $0.596(0.428-0.831)$ & 0.002 & $0.572(0.37 I-0.882)$ & 0.012 \\
\hline hsa-mir-3I (high vs low) & 1.559 (1.109-2.191) & 0.011 & $2.000(1.286-3.1 \mathrm{II})$ & 0.002 \\
\hline
\end{tabular}

Note: aStatistical significant results in bold.

Abbreviations: $\mathrm{Cl}$, confidence interval; $\mathrm{HR}$, hazard ratio.

family (mir-200a, mir-200b, and mir-429) was shown to play a critical role in the progression of LUAD. Finally, these 185 differentially expressed miRNAs were subjected to univariate and multivariate Cox proportional hazard regression analyses. Four (mir-1468, mir-212, mir-3653, and mir-31) and three (mir-551b, mir-3653, and mir-31) miRNAs were validated as independent predictors for RFS and OS of LUAD patients, respectively. Overall, the findings of this study can be a complement to the previous studies. ${ }^{9}, 12,14$

mir-200 family consisting of five (mir-200a, mir-200b, mir-200c, mir-141, and mir-429) miRNAs acts as either oncogenes or tumor suppressors in various human cancers and is significantly involved in the processes of epithelialto-mesenchymal transition, cell invasion, and metastasis. ${ }^{24-27}$ Based on the chromosomal locations, the mir-200 family can be divided into two clusters. One is the mir-200ba/429 cluster containing mir-200a, mir-200b, and mir- 429 , which is located on chromosome $1 \mathrm{p} 36 .{ }^{28}$ This study has demonstrated that the mir-200ba/429 cluster was significantly associated with three pathologic variables (pathologic $\mathrm{T}$, pathologic N, and pathologic Stage) in LUAD. Interestingly, upregulated expression levels of these three miRNAs were observed between tumor and normal tissues, which indicated the oncogene role of this cluster. Inversely, this cluster declined during tumor progression acting as the tumor suppressor. As a previous study reported, ${ }^{29}$ these three miRNAs could be expressed in a wavelike manner during carcinogenesis. Specifically, these three miRNAs were upregulated in tumor initiation but possibly downregulated during tumor progression. In addition, it has been noted that low expression level of mir-200 family could predict poor survival and serve as a prognostic marker for various cancers. ${ }^{30,31}$ Unfortunately, this study failed to elucidate the prognostic role of the mir-200ba/429 cluster in LUAD.

Table 3 Univariate and multivariate Cox regression analyses of overall survival in lung adenocarcinoma

\begin{tabular}{|c|c|c|c|c|}
\hline \multirow[t]{2}{*}{ Variables } & \multicolumn{2}{|l|}{ Univariate analysis } & \multicolumn{2}{|c|}{ Multivariate analysis } \\
\hline & HR (95\% CI) & $P$-value ${ }^{a}$ & HR (95\% CI) & $P$-value ${ }^{a}$ \\
\hline Age ( $\geq 65$ years vs $<65$ years) & $0.990(0.711-1.377)$ & 0.951 & & \\
\hline Sex (male vs female) & $0.918(0.66 I-1.275)$ & 0.610 & & \\
\hline $\mathrm{T}(\mathrm{T} 3+\mathrm{T} 4 \mathrm{vs} \mathrm{TI}+\mathrm{T} 2)$ & $2.372(1.552-3.623)$ & 0.000 & $2.617(1.406-4.871)$ & 0.002 \\
\hline $\mathrm{N}(\mathrm{N} I+\mathrm{N} 2+\mathrm{N} 3$ vs N0) & $2.555(1.833-3.56 I)$ & 0.000 & & \\
\hline M (MI vs M0) & $2.342(1.308-4.194)$ & 0.004 & & \\
\hline Stage (III + IV vs I + II) & $2.615(1.853-3.691)$ & 0.000 & $2.004(I .195-3.36 I)$ & 0.008 \\
\hline hsa-mir-548v (high vs low) & $0.589(0.415-0.835)$ & 0.003 & & \\
\hline hsa-mir-30e (high vs low) & $0.681(0.490-0.945)$ & 0.022 & & \\
\hline hsa-mir-I468 (high vs low) & $0.668(0.478-0.933)$ & 0.018 & & \\
\hline hsa-mir-55Ib (high vs low) & $0.646(0.457-0.913)$ & 0.013 & $0.550(0.346-0.874)$ & 0.011 \\
\hline hsa-mir-2I 2 (high vs low) & I.50| (I.080-2.086) & 0.016 & & \\
\hline hsa-mir-3607 (high vs low) & $0.666(0.478-0.927)$ & 0.016 & & \\
\hline hsa-mir-3653 (high vs low) & $0.559(0.40 \mathrm{I}-0.780)$ & 0.001 & $0.562(0.35 \mathrm{I}-0.899)$ & 0.016 \\
\hline hsa-mir-3I (high vs low) & 1.544 (I.097-2.172) & 0.013 & $2.181(1.382-3.440)$ & 0.001 \\
\hline
\end{tabular}

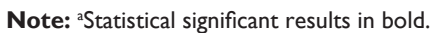

Abbreviations: $\mathrm{Cl}$, confidence interval; $\mathrm{HR}$, hazard ratio. 

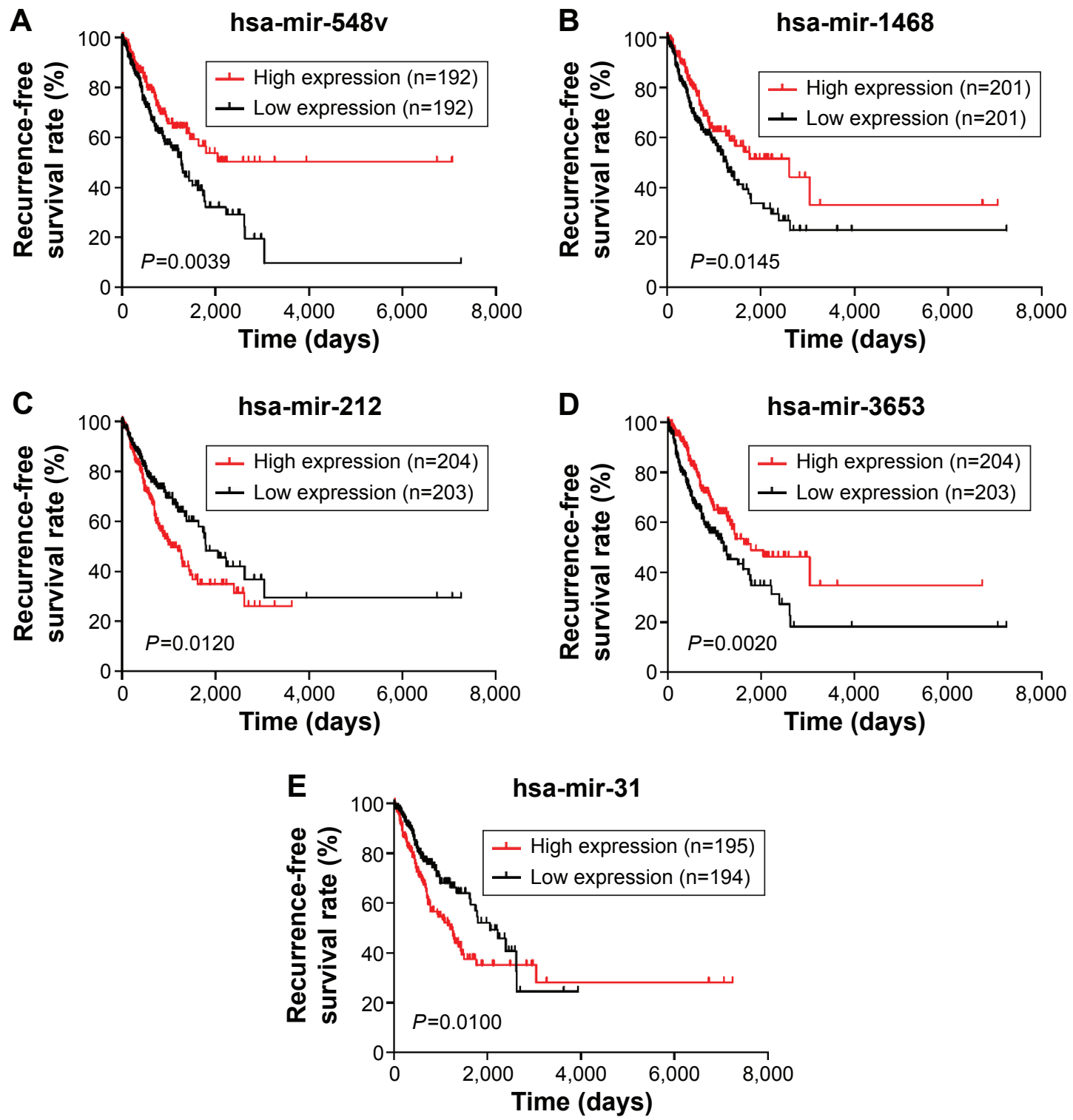

Figure 3 Kaplan-Meier survival curves for recurrence-free survival of lung adenocarcinoma patients. Notes: (A) hsa-mir-548v; (B) hsa-mir-1468; (C) hsa-mir-2I2; (D) hsa-mir-3653; and (E) hsa-mir-3I.

Among the five survival-related miRNAs, two miRNAs (mir-212 and mir-31) were negative prognosticators and the remaining three miRNAs (mir-551b, mir-1468, and mir3653) were positive prognosticators according to the association between their expression level and patient survival. With regard to the two risky miRNAs, the role of mir-212 remains to be controversial in lung cancer. Li et $\mathrm{al}^{32}$ found that mir212 displayed tumor-promoting properties by targeting the Hedgehog signaling pathway in non-small-cell lung cancer. In contrast, Incoronato et $\mathrm{a}^{33}$ revealed the tumor suppressor role of mir-212 by promoting cancer cell apoptosis in nonsmall-cell lung cancer. However, the findings of this study were in-line with the former. Upregulated mir-212 expression was identified in the comparison between LUAD and adjacent normal tissues, and the highly expressed mir-212 was associated with poor RFS. Meng et $\mathrm{al}^{13}$ showed that mir-31 increased cell migration, invasion, and proliferation in an ERK1/2 signaling-dependent manner, and the low expressed mir-31 predicted excellent survival. Furthermore, a recent meta-analysis confirmed that high mir-31 expression was associated with poor survival in general cancers, and mir-31 may be a useful clinical prognostic biomarker. ${ }^{35}$ Fortunately, mir-31 predicted poor RFS and OS in this study, which was in agreement with previous studies. Regarding the protective miRNAs, to the authors' knowledge, this is the first time to report the association of mir-551b, mir-1468, and mir-3653 with OS or RFS in LUAD, and the functions of these three miRNAs in cancers were poorly understood. 

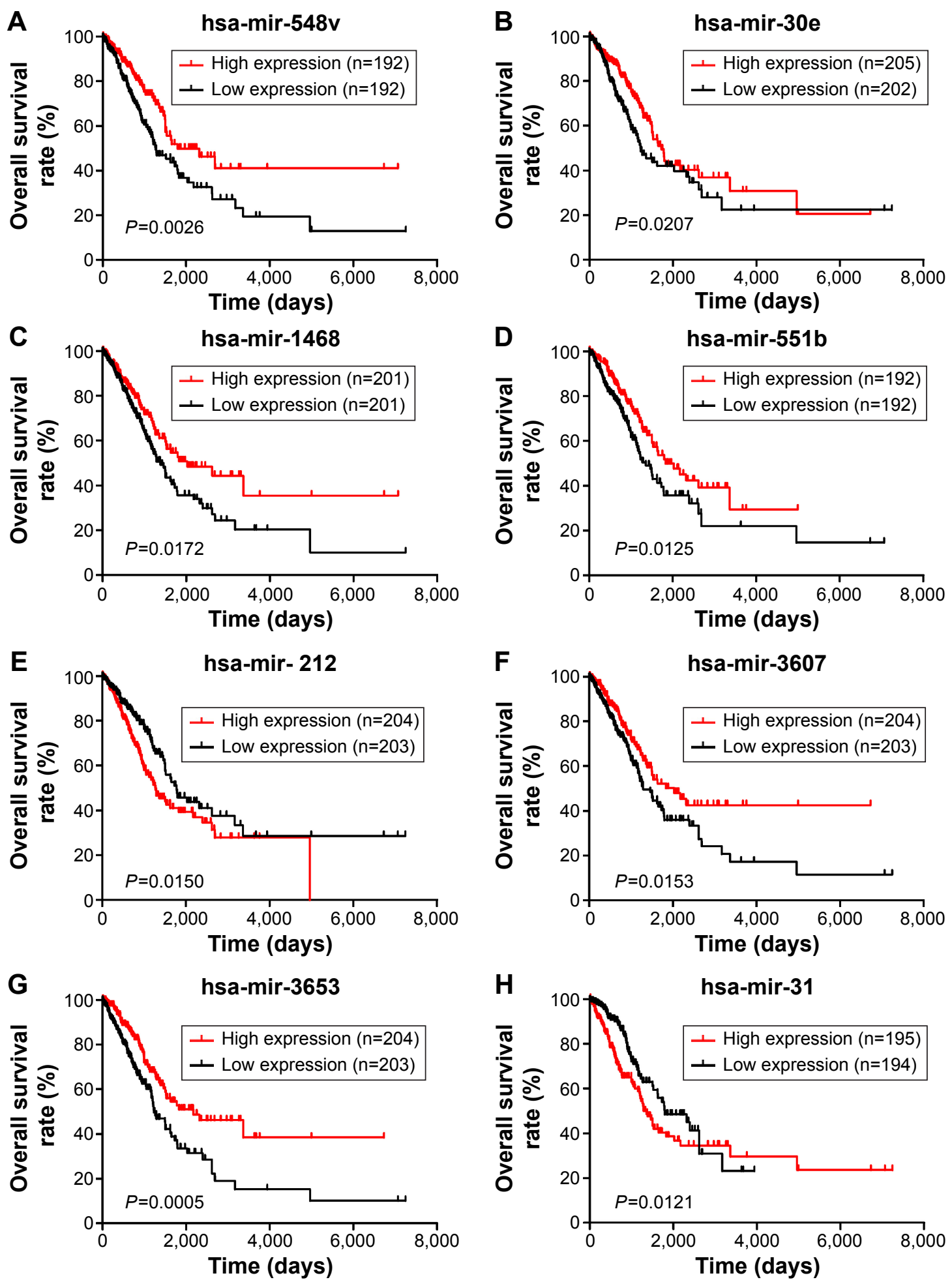

Figure 4 Kaplan-Meier survival curves for overall survival of lung adenocarcinoma patients.

Notes: (A) hsa-mir-548v; (B) hsa-mir-30e; (C) hsa-mir-I468; (D) hsa-mir-55Ib; (E) hsa-mir-2I2; (F) hsa-mir-3607; (G) hsa-mir-3653; and (H) hsa-mir-3I.

\section{Limitations}

However, several limitations in this study should be noted. First, the majority of patients enrolled in this study were Caucasians and subjects from Asia accounted for only 1.4\%, which affected the generalizability of the findings of the study. Additional sets with samples from non-Caucasians will be needed to confirm these findings. Second, only 1,046 human miRNAs were analyzed. So, miRNAs identified here may not represent all candidates that associated with tumor progression and prognosis of LUAD. Third, the follow-up 
data downloaded from TCGA had a relatively high censored rate, which may influence the survival analysis.

\section{Conclusion}

This study identified 185 differentially expressed miRNAs between LUAD tumor tissues and adjacent normal tissues, and further confirmed the progression-related and prognosis-related miRNAs that may show the underlying molecular mechanism and predict the prognosis of LUAD. Given that false-positive results do potentially exist, these findings require further validation with large-scale independent cohorts.

\section{Acknowledgments}

The authors thank Doctor Houqun Ying, Ms Jie Chen, and Ms Xian Liu for their statistic and technical assistance. This project was supported by grants from the National Nature Science Foundation of China (numbers 81472027 and 81200401), Nanjing Science and Technology Committee Project (number 201108025), Nanjing Medical Technology Development Project (number ZKX11025), Nanjing Health Young Talent Project, and Jiangsu Provincial Key Medical Talents to SKW; Nanjing Medical Science and Technique Development Foundation to YQP (number QRX11255) and BSH (number QRX11254); and Nanjing Medical University Science and Technique Development Foundation Project to HLS (number 2015NJMUZD049).

\section{Disclosure}

The authors report no conflicts of interest in this work.

\section{References}

1. Torre LA, Bray F, Siegel RL, Ferlay J, Lortet-Tieulent J, Jemal A. Global cancer statistics, 2012. CA Cancer J Clin. 2015;65(2):87-108.

2. Chen W, Zheng R, Baade PD, et al. Cancer statistics in China, 2015. CA Cancer J Clin. 2016;66(2):115-132.

3. Siegel RL, Miller KD, Jemal A. Cancer statistics, 2016. $C A$ Cancer J Clin. 2016;66(1):7-30.

4. Devarakonda S, Morgensztern D, Govindan R. Genomic alterations in lung adenocarcinoma. Lancet Oncol. 2015;16(7):e342-e351.

5. Ringner M, Jonsson G, Staaf J. Prognostic and chemotherapy predictive value of gene-expression phenotypes in primary lung adenocarcinoma. Clin Cancer Res. 2016;22(1):218-229.

6. Bartel DP. MicroRNAs: genomics, biogenesis, mechanism, and function. Cell. 2004;116(2):281-297.

7. Iorio MV, Croce CM. MicroRNA involvement in human cancer. Carcinogenesis. 2012;33(6):1126-1133.

8. Jansson MD, Lund AH. MicroRNA and cancer. Mol Oncol. 2012;6(6): 590-610.

9. Yu SL, Chen HY, Chang GC, et al. MicroRNA signature predicts survival and relapse in lung cancer. Cancer Cell. 2008;13(1):48-57.

10. Gao Y, Xue Q, Wang D, Du M, Zhang Y, Gao S. miR-873 induces lung adenocarcinoma cell proliferation and migration by targeting SRCIN1. Am J Transl Res. 2015;7(11):2519-2526.
11. Qu J, Li M, An J, et al. MicroRNA-33b inhibits lung adenocarcinoma cell growth, invasion, and epithelial-mesenchymal transition by suppressing Wnt/beta-catenin/ZEB1 signaling. Int J Oncol. 2015;47(6): 2141-2152.

12. Li X, Shi Y, Yin Z, Xue X, Zhou B. An eight-miRNA signature as a potential biomarker for predicting survival in lung adenocarcinoma. J Transl Med. 2014;12:159.

13. Edmonds MD, Eischen CM. Differences in miRNA expression in early stage lung adenocarcinomas that did and did not relapse. PLoS One. 2014;9(7):e101802.

14. Yan G, Yao R, Tang D, et al. Prognostic significance of microRNA expression in completely resected lung adenocarcinoma and the associated response to erlotinib. Med Oncol. 2014;31(10):203.

15. Hong JH, Roh KS, Suh SS, et al. The expression of microRNA-34a is inversely correlated with c-MET and CDK6 and has a prognostic significance in lung adenocarcinoma patients. Tumour Biol. 2015;36(12):9327-9337.

16. Yanaihara N, Caplen N, Bowman E, et al. Unique microRNA molecular profiles in lung cancer diagnosis and prognosis. Cancer Cell. 2006;9(3):189-198.

17. Cancer Genome Atlas Research Network. Comprehensive molecular profiling of lung adenocarcinoma. Nature. 2014;511(7511):543-550.

18. Zhao Y, Simon R. BRB-ArrayTools Data Archive for human cancer gene expression: a unique and efficient data sharing resource. Cancer Inform. 2008;6:9-15.

19. Calin GA, Croce CM. MicroRNA signatures in human cancers. Nat Rev Cancer. 2006;6(11):857-866.

20. Lu J, Getz G, Miska EA, et al. MicroRNA expression profiles classify human cancers. Nature. 2005;435(7043):834-838.

21. Zhang J, Chong CC, Chen GG, Lai PB. A seven-microRNA expression signature predicts survival in hepatocellular carcinoma. PLoS One. 2015;10(6):e0128628

22. Ge YZ, Xu LW, Xu Z, et al. Expression profiles and clinical significance of MicroRNAs in papillary renal cell carcinoma: a STROBE-compliant observational study. Medicine (Baltimore). 2015;94(16):e767.

23. Xu Z, Yu YQ, Ge YZ, et al. MicroRNA expression profiles in muscle-invasive bladder cancer: identification of a four-microRNA signature associated with patient survival. Tumour Biol. 2015;36(10): 8159-8166.

24. Korpal M, Kang Y. The emerging role of miR-200 family of microRNAs in epithelial-mesenchymal transition and cancer metastasis. RNA Biol. 2008;5(3):115-119.

25. Mongroo PS, Rustgi AK. The role of the miR-200 family in epithelialmesenchymal transition. Cancer Biol Ther. 2010;10(3):219-222.

26. Huang XY, Yao JG, Huang HD, et al. MicroRNA-429 modulates hepatocellular carcinoma prognosis and tumorigenesis. Gastroenterol Res Pract. 2013;2013:804128.

27. Li J, Du L, Yang Y, et al. MiR-429 is an independent prognostic factor in colorectal cancer and exerts its anti-apoptotic function by targeting SOX2. Cancer Lett. 2013;329(1):84-90.

28. Feng X, Wang Z, Fillmore R, Xi Y. MiR-200, a new star miRNA in human cancer. Cancer Lett. 2014;344(2):166-173.

29. Meng X, Joosse SA, Muller V, et al. Diagnostic and prognostic potential of serum miR-7, miR-16, miR-25, miR-93, miR-182, miR-376a and miR-429 in ovarian cancer patients. Br J Cancer. 2015;113(9): 1358-1366.

30. $\mathrm{Hu} \mathrm{X}$, Macdonald DM, Huettner PC, et al. A miR-200 microRNA cluster as prognostic marker in advanced ovarian cancer. Gynecol Oncol. 2009;114(3):457-464.

31. Pichler M, Ress AL, Winter E, et al. MiR-200a regulates epithelial to mesenchymal transition-related gene expression and determines prognosis in colorectal cancer patients. Br J Cancer. 2014;110(6): $1614-1621$

32. Li Y, Zhang D, Chen C, Ruan Z, Li Y, Huang Y. MicroRNA-212 displays tumor-promoting properties in non-small cell lung cancer cells and targets the hedgehog pathway receptor PTCH1. Mol Biol Cell. 2012; 23(8):1423-1434. 
33. Incoronato M, Garofalo M, Urso L, et al. miR-212 increases tumor necrosis factor-related apoptosis-inducing ligand sensitivity in nonsmall cell lung cancer by targeting the antiapoptotic protein PED Cancer Res. 2010;70(9):3638-3646.

34. Meng W, Ye Z, Cui R, et al. MicroRNA-31 predicts the presence of lymph node metastases and survival in patients with lung adenocarcinoma. Clin Cancer Res. 2013;19(19):5423-5433.
35. Wang S, Hu J, Zhang D, Li J, Fei Q, Sun Y. Prognostic role of microRNA-31 in various cancers: a meta-analysis. Tumour Biol. 2014; 35(11):11639-11645 


\section{Supplementary materials}

Table SI Differentially expressed microRNAs between lung adenocarcinoma and adjacent normal tissues

\begin{tabular}{|c|c|c|c|c|c|}
\hline \multirow[t]{2}{*}{ MicroRNA } & \multicolumn{2}{|c|}{ Expression level $^{\mathrm{a}}$} & \multirow{2}{*}{$\begin{array}{l}\text { Fold } \\
\text { change }\end{array}$} & \multirow[t]{2}{*}{$P$-value } & \multirow[t]{2}{*}{ FDR } \\
\hline & LUAD & Normal & & & \\
\hline hsa-mir-210 & $9.54 \pm 1.69$ & $4.62 \pm 1.58$ & 30.17 & $<\mathrm{Ie}-07$ & $<\mathrm{Ie}-07$ \\
\hline hsa-mir-9-I & $8.68 \pm 2.36$ & $4.38 \pm 1.97$ & 19.81 & $<\mathrm{le}-07$ & $<$ Ie-07 \\
\hline hsa-mir-9-2 & $8.67 \pm 2.35$ & $4.39 \pm 2.09$ & 19.47 & $<$ le-07 & $<$ Ie-07 \\
\hline hsa-mir-196a-I & $3.76 \pm 3.39$ & $0.14 \pm 1.86$ & 12.33 & $<\mathrm{Ie}-07$ & $<$ Ie-07 \\
\hline hsa-mir-3607 & $4.73 \pm 1.86$ & $1.38 \pm 1.87$ & 10.21 & $<\mathrm{Ie}-07$ & $<$ Ie-07 \\
\hline hsa-mir-I53-2 & $3.33 \pm 1.74$ & $0.23 \pm 1.27$ & 8.61 & $<\mathrm{Ie}-07$ & $<$ le-07 \\
\hline hsa-mir-3I & $2.39 \pm 2.71$ & $-0.66 \pm 1.68$ & 8.32 & 0.0000001 & 0.000000391 \\
\hline hsa-mir-182 & $14.35 \pm 0.92$ & $11.58 \pm 1.48$ & 6.84 & $<\mathrm{le}-07$ & $<\mathrm{le}-07$ \\
\hline hsa-mir-96 & $4.46 \pm I .1 I$ & $1.72 \pm 1.42$ & 6.67 & $<\mathrm{le}-07$ & $<$ Ie-07 \\
\hline hsa-mir-708 & $6.52 \pm 1.26$ & $3.83 \pm 1.43$ & 6.45 & $<$ le-07 & $<$ Ie-07 \\
\hline hsa-mir-I 42 & $\mathrm{II} .14 \pm 1.26$ & $8.47 \pm 2.21$ & 6.36 & $<\mathrm{Ie}-07$ & $<\mathrm{le}-07$ \\
\hline hsa-mir-I35b & $5.54 \pm 1.7 \mid$ & $2.88 \pm 1.75$ & 6.29 & $<\mathrm{Ie}-07$ & $<\mathrm{Ie}-07$ \\
\hline hsa-mir-I 83 & $13.17 \pm 1.06$ & $10.66 \pm 0.59$ & 5.71 & $<$ Ie-07 & $<$ Ie-07 \\
\hline hsa-mir-33b & $\mathrm{I} .72 \pm \mathrm{I} .44$ & $-0.75 \pm 1.25$ & 5.53 & $<$ Ie-07 & $<$ Ie-07 \\
\hline hsa-mir-|4| & $|0.54 \pm 0.9|$ & $8.15 \pm 1.92$ & 5.23 & $<\mathrm{le}-07$ & $<\mathrm{le}-07$ \\
\hline hsa-mir-503 & $2.74 \pm \mathrm{I} .4 \mathrm{I}$ & $0.37 \pm 1.13$ & 5.17 & $<\mathrm{Ie}-07$ & $<\mathrm{Ie}-07$ \\
\hline hsa-mir-6I6 & $1.34 \pm 1.43$ & $-0.99 \pm 1.14$ & 5.02 & $<\mathrm{le}-07$ & $<$ le-07 \\
\hline hsa-mir-I48a & $15.36 \pm 0.98$ & $13.15 \pm 1.06$ & 4.63 & $<$ Ie-07 & $<\mathrm{le}-07$ \\
\hline hsa-mir-30la & $3.61 \pm 1.27$ & $1.44 \pm 1.21$ & 4.5 & $<\mathrm{le}-07$ & $<$ le-07 \\
\hline hsa-mir-200a & $9.62 \pm 1.07$ & $7.51 \pm 1.03$ & 4.34 & $<\mathrm{le}-07$ & $<$ Ie-07 \\
\hline hsa-mir-345 & $3.94 \pm 1.21$ & $\mathrm{I} .87 \pm \mathrm{I} .27$ & 4.2 & $<\mathrm{le}-07$ & $<$ Ie-07 \\
\hline hsa-mir-29b-I & $8.66 \pm 0.99$ & $6.66 \pm 2.01$ & 3.99 & $<$ le-07 & $<\mathrm{le}-07$ \\
\hline hsa-mir-205 & $6.39 \pm 2.92$ & $4.4 I \pm 2.62$ & 3.97 & 0.0002091 & 0.000506 \\
\hline hsa-mir-196b & $4.66 \pm 2.47$ & $2.68 \pm 1.59$ & 3.95 & 0.0000099 & 0.0000293 \\
\hline hsa-mir-323b & $0.9 \pm 2.04$ & $-1.07 \pm 1.59$ & 3.92 & 0.0000997 & 0.000253 \\
\hline hsa-mir-7-I & $4.3 \pm 0.88$ & $2.44 \pm 1.67$ & 3.62 & $<\mathrm{le}-07$ & $<\mathrm{le}-07$ \\
\hline hsa-mir-429 & $6.73 \pm 1.21$ & $4.94 \pm 2.15$ & 3.44 & $<\mathrm{le}-07$ & $<\mathrm{le}-07$ \\
\hline hsa-mir-937 & $1.82 \pm 1.82$ & $0.07 \pm 1.57$ & 3.36 & 0.0000005 & 0.0000018 \\
\hline hsa-mir-192 & $9.13 \pm 2$ & $7.43 \pm 1.84$ & 3.24 & 0.0000044 & 0.0000135 \\
\hline hsa-mir-539 & $1.35 \pm 1.68$ & $-0.3 \pm 1.14$ & 3.15 & 0.0000017 & 0.00000567 \\
\hline hsa-mir-89la & $1.42 \pm 2.26$ & $-0.23 \pm 1.9$ & 3.15 & 0.0005067 & 0.00115 \\
\hline hsa-mir-33a & $4.19 \pm 1.23$ & $2.56 \pm 1.07$ & 3.09 & $<\mathrm{le}-07$ & $<$ Ie-07 \\
\hline hsa-mir-20a & $7.63 \pm 0.97$ & $6.01 \pm 2.2$ & 3.08 & $<$ le-07 & $<$ le-07 \\
\hline hsa-mir-I30b & $4.92 \pm 1.03$ & $3.35 \pm 0.81$ & 2.96 & $<\mathrm{le}-07$ & $<$ le-07 \\
\hline hsa-mir-3648 & $0.9 \pm 1.34$ & $-0.66 \pm 1.63$ & 2.93 & $<\mathrm{le}-07$ & $<\mathrm{le}-07$ \\
\hline hsa-mir-548v & $0.59 \pm 1.5$ & $-0.95 \pm 1.11$ & 2.91 & 0.0000028 & 0.00000906 \\
\hline hsa-mir-199b & $11.06 \pm 0.99$ & $9.57 \pm 1.09$ & 2.81 & $<\mathrm{le}-07$ & $<\mathrm{le}-07$ \\
\hline hsa-mir-450a-I & $1.75 \pm 1.46$ & $0.27 \pm 1.17$ & 2.78 & 0.0000004 & 0.00000147 \\
\hline hsa-mir-424 & $6.19 \pm 1.24$ & $4.73 \pm 1.09$ & 2.76 & $<\mathrm{le}-07$ & $<\mathrm{le}-07$ \\
\hline hsa-mir-29b-2 & $8.8 \pm 0.97$ & $7.34 \pm 0.99$ & 2.74 & $<\mathrm{Ie}-07$ & $<\mathrm{le}-07$ \\
\hline hsa-mir- 187 & $3.63 \pm 2.3$ & $2.17 \pm 1.53$ & 2.74 & 0.0005938 & 0.00132 \\
\hline hsa-mir-2355 & $5.78 \pm 1.04$ & $4.33 \pm 2$ & 2.73 & $<\mathrm{le}-07$ & $<\mathrm{le}-07$ \\
\hline hsa-mir-675 & $2.56 \pm 2.66$ & $1.12 \pm 2.05$ & 2.7 & 0.0035632 & 0.00664 \\
\hline hsa-mir-455 & $7.19 \pm 1.08$ & $5.77 \pm 1.32$ & 2.68 & $<\mathrm{le}-07$ & $<\mathrm{le}-07$ \\
\hline hsa-mir-224 & $4.97 \pm 1.86$ & $3.55 \pm 0.99$ & 2.68 & 0.0000233 & 0.0000648 \\
\hline hsa-mir-193b & $5.69 \pm 1.4$ & $4.28 \pm 1.22$ & 2.67 & $<\mathrm{le}-07$ & $<\mathrm{le}-07$ \\
\hline hsa-mir-450a-2 & $1.7 \pm 1.48$ & $0.29 \pm 1.2$ & 2.66 & 0.0000017 & 0.00000567 \\
\hline hsa-mir-642a & $\mathrm{I} .84 \pm \mathrm{I} .4$ & $0.46 \pm 2.12$ & 2.6 & 0.0000008 & 0.00000282 \\
\hline hsa-mir-103-2 & $3.19 \pm 0.8$ & $1.85 \pm 1.35$ & 2.54 & $<\mathrm{le}-07$ & $<\mathrm{le}-07$ \\
\hline hsa-mir-93 & $11.78 \pm 0.98$ & $10.45 \pm 0.49$ & 2.52 & $<\mathrm{le}-07$ & $<$ le-07 \\
\hline
\end{tabular}


Table SI (Continued)

\begin{tabular}{|c|c|c|c|c|c|}
\hline \multirow[t]{2}{*}{ MicroRNA } & \multicolumn{2}{|c|}{ Expression level ${ }^{a}$} & \multirow{2}{*}{$\begin{array}{l}\text { Fold } \\
\text { change }\end{array}$} & \multirow[t]{2}{*}{$P$-value } & \multirow[t]{2}{*}{ FDR } \\
\hline & LUAD & Normal & & & \\
\hline hsa-mir-I307 & $10.31 \pm 0.92$ & $8.98 \pm 1.53$ & 2.5 & $<\mathrm{le}-07$ & $<\mathrm{le}-07$ \\
\hline hsa-mir-409 & $3.76 \pm 1.54$ & $2.45 \pm 1.25$ & 2.48 & 0.0000034 & 0.0000108 \\
\hline hsa-mir-18Ib-2 & $2.54 \pm 1.09$ & $1.24 \pm 1.06$ & 2.46 & $<\mathrm{le}-07$ & $<\mathrm{le}-07$ \\
\hline hsa-mir-19b-I & $2.65 \pm 1$ & $1.35 \pm 1.31$ & 2.45 & $<\mathrm{le}-07$ & $<\mathrm{le}-07$ \\
\hline hsa-mir-199a-I & $9.89 \pm 0.94$ & $8.6 \pm 0.8$ & 2.44 & $<\mathrm{le}-07$ & $<\mathrm{le}-07$ \\
\hline hsa-mir-199a-2 & $10.66 \pm 0.94$ & $9.37 \pm 0.82$ & 2.44 & $<\mathrm{le}-07$ & $<\mathrm{le}-07$ \\
\hline hsa-mir-। 88 & $1.24 \pm 1.19$ & $-0.03 \pm 1.3$ & 2.42 & $<\mathrm{le}-07$ & $<\mathrm{le}-07$ \\
\hline hsa-mir-940 & $\mathrm{I} .04 \pm \mathrm{I} .4 \mathrm{I}$ & $-0.23 \pm 1.58$ & 2.42 & 0.0000032 & 0.0000102 \\
\hline hsa-mir-I30I & $3.68 \pm 1.13$ & $2.47 \pm 1.18$ & 2.32 & $<\mathrm{le}-07$ & $<\mathrm{Ie}-07$ \\
\hline hsa-mir-542 & $7.77 \pm 1.21$ & $6.55 \pm 1.75$ & 2.32 & 0.0000002 & 0.000000755 \\
\hline hsa-mir-323 & $0.54 \pm 1.9$ & $-0.65 \pm 1.45$ & 2.28 & 0.0039901 & 0.00719 \\
\hline hsa-mir-194-I & $6.77 \pm 1.88$ & $5.59 \pm 1.83$ & 2.27 & 0.0006354 & 0.0014 \\
\hline hsa-mir-19b-2 & $6.5 \pm 0.88$ & $5.35 \pm 2.38$ & 2.23 & $<\mathrm{le}-07$ & $<\mathrm{le}-07$ \\
\hline hsa-mir-26a-I & $0.22 \pm 1.26$ & $-0.94 \pm 1.04$ & 2.22 & 0.0000063 & 0.0000192 \\
\hline hsa-mir-760 & $0.77 \pm 1.54$ & $-0.38 \pm 1.45$ & 2.21 & 0.0001207 & 0.000301 \\
\hline hsa-mir-425 & $7.4 \pm 0.85$ & $6.27 \pm 0.71$ & 2.2 & $<\mathrm{le}-07$ & $<\mathrm{le}-07$ \\
\hline hsa-mir-550a-I & $1.69 \pm 1.16$ & $0.58 \pm 1.43$ & 2.16 & 0.0000006 & 0.00000214 \\
\hline hsa-mir-34a & $7.65 \pm 0.88$ & $6.54 \pm 0.92$ & 2.15 & $<\mathrm{le}-07$ & $<\mathrm{le}-07$ \\
\hline hsa-mir-550a-2 & $1.32 \pm 1.19$ & $0.23 \pm 1.27$ & 2.13 & 0.0000015 & 0.00000517 \\
\hline hsa-mir-628 & $3.9 \pm 1.12$ & $2.82 \pm 1.66$ & 2.12 & 0.0000011 & 0.00000383 \\
\hline hsa-mir-I 36 & $4.27 \pm 1.42$ & $3.19 \pm 1.33$ & 2.11 & 0.000042 & 0.000111 \\
\hline hsa-mir-106a & $3.42 \pm 1.31$ & $2.35 \pm 1.9$ & 2.09 & 0.0000374 & 0.0000996 \\
\hline hsa-mir-55Ib & $0.99 \pm 1.89$ & $-0.06 \pm 1.44$ & 2.08 & 0.006576 & 0.0115 \\
\hline hsa-mir-194-2 & $6.93 \pm 1.9$ & $5.89 \pm 1.8$ & 2.06 & 0.0027277 & 0.0053 \\
\hline hsa-mir-939 & $-0.09 \pm 1.16$ & $-1.12 \pm 1$ & 2.05 & 0.0000155 & 0.0000447 \\
\hline hsa-mir-629 & $6.83 \pm 0.92$ & $5.8 I \pm 0.68$ & 2.04 & $<\mathrm{le}-07$ & $<\mathrm{le}-07$ \\
\hline hsa-mir-200b & $9.26 \pm 1.08$ & $8.24 \pm 1.09$ & 2.04 & 0.0000003 & 0.00000112 \\
\hline hsa-mir-625 & $7.93 \pm 0.94$ & $6.93 \pm 0.7$ & 2.01 & $<\mathrm{le}-07$ & $<\mathrm{le}-07$ \\
\hline hsa-mir-592 & $0.9 \pm 1.42$ & $-0.09 \pm 1.4$ & 1.99 & 0.0018302 & 0.00372 \\
\hline hsa-mir-I7 & $9.34 \pm 0.84$ & $8.35 \pm 1.26$ & 1.98 & $<\mathrm{le}-07$ & $<\mathrm{le}-07$ \\
\hline hsa-mir-22 & $16.17 \pm 0.65$ & $15.19 \pm 0.84$ & 1.97 & $<\mathrm{le}-07$ & $<\mathrm{le}-07$ \\
\hline hsa-mir-766 & $3.06 \pm 1.12$ & $2.08 \pm 1.91$ & 1.97 & 0.0000103 & 0.0000302 \\
\hline hsa-mir-375 & $|4.04 \pm 1.8|$ & $13.06 \pm 1.3$ & 1.97 & 0.0027744 & 0.00536 \\
\hline hsa-mir-454 & $2.89 \pm 0.87$ & $1.93 \pm 1.23$ & 1.95 & $<\mathrm{le}-07$ & $<\mathrm{le}-07$ \\
\hline hsa-mir-3I27 & $2.3 \pm 1.21$ & $1.35 \pm 1.29$ & 1.93 & 0.0000253 & 0.0000685 \\
\hline hsa-mir-3676 & $1.39 \pm 1.6$ & $0.44 \pm 1.39$ & 1.93 & 0.0019053 & 0.00382 \\
\hline hsa-mir-3200 & $\mathrm{I} .4 \mathrm{I} \pm \mathrm{I} .57$ & $0.47 \pm 1.41$ & 1.92 & 0.0013135 & 0.00278 \\
\hline hsa-mir-33I & $5.39 \pm 0.93$ & $4.46 \pm 1.13$ & 1.91 & 0.0000001 & 0.000000391 \\
\hline hsa-mir-493 & $2.9 \pm 1.46$ & $1.97 \pm 1.15$ & 1.9 & 0.0005135 & 0.00115 \\
\hline hsa-mir-660 & $5.28 \pm I . I$ & $4.36 \pm 2.66$ & 1.89 & 0.000098 & $0.00025 I$ \\
\hline hsa-mir-2I 7 & $4.08 \pm 1.63$ & $3.15 \pm 1.94$ & 1.89 & 0.0032523 & 0.00614 \\
\hline hsa-mir-590 & $4.01 \pm 0.96$ & $3.1 \pm 0.75$ & 1.88 & 0.0000018 & 0.00000594 \\
\hline hsa-mir-576 & $3.08 \pm 0.98$ & $2.19 \pm 1.34$ & 1.86 & 0.0000017 & 0.00000567 \\
\hline hsa-mir-19a & $4.13 \pm 1.18$ & $3.26 \pm 1.22$ & 1.84 & 0.0001625 & 0.000396 \\
\hline hsa-mir-320b-2 & $1.9 \pm 1.17$ & $1.04 \pm 1.06$ & 1.82 & 0.0000604 & 0.000157 \\
\hline hsa-mir-374a & $9.39 \pm 0.87$ & $8.53 \pm 3.24$ & 1.82 & 0.0001007 & 0.000253 \\
\hline hsa-mir-382 & $3.27 \pm 1.5$ & $2.4 I \pm I .38$ & 1.82 & 0.0016885 & 0.00345 \\
\hline hsa-mir-487b & $1.47 \pm 1.61$ & $0.61 \pm 1.35$ & 1.82 & 0.0036683 & 0.0068 \\
\hline hsa-mir-582 & $7.83 \pm 1.61$ & $6.97 \pm 2.27$ & 1.82 & 0.0046804 & 0.00834 \\
\hline hsa-mir-191 & $8.76 \pm 0.71$ & $7.9 \pm 0.78$ & 1.81 & $<\mathrm{le}-07$ & $<\mathrm{le}-07$ \\
\hline hsa-mir-I 287 & $5.7 \pm 1.06$ & $4.84 \pm 0.8$ & 1.81 & 0.0000097 & 0.000029 \\
\hline hsa-mir-|27| & $0.74 \pm I .21$ & $-0.1 \mathrm{I} \pm \mathrm{I} .14$ & 1.8 & 0.0002572 & 0.000608 \\
\hline hsa-mir-3647 & $2.7 I \pm I .24$ & $1.87 \pm 1.72$ & 1.79 & 0.0003578 & 0.000834 \\
\hline
\end{tabular}


Table SI (Continued)

\begin{tabular}{|c|c|c|c|c|c|}
\hline \multirow[t]{2}{*}{ MicroRNA } & \multicolumn{2}{|c|}{ Expression level $^{a}$} & \multirow{2}{*}{$\begin{array}{l}\text { Fold } \\
\text { change }\end{array}$} & \multirow[t]{2}{*}{$P$-value } & \multirow[t]{2}{*}{ FDR } \\
\hline & LUAD & Normal & & & \\
\hline hsa-mir-128-2 & $5.79 \pm 1.02$ & $4.97 \pm 1.24$ & 1.76 & 0.0000252 & 0.0000685 \\
\hline hsa-mir-67I & $2.77 \pm 0.88$ & $1.98 \pm 1.3$ & 1.73 & 0.000004 & 0.0000124 \\
\hline hsa-mir-3677 & $2.19 \pm 1.4$ & $1.42 \pm 1.07$ & 1.71 & $0.0037|5|$ & 0.00685 \\
\hline hsa-mir-I5a & $6.97 \pm 0.72$ & $6.2 \pm 2.07$ & 1.7 & 0.0000025 & 0.00000817 \\
\hline hsa-mir-3653 & $2.49 \pm 1.1$ & $1.73 \pm 1.84$ & 1.7 & 0.0004719 & 0.00108 \\
\hline hsa-mir-3605 & $1.15 \pm 1.02$ & $0.4 \pm 1.85$ & 1.68 & 0.0002559 & 0.000608 \\
\hline hsa-mir-18a & $3.44 \pm 1.2$ & $2.72 \pm 1.61$ & 1.66 & 0.0013779 & 0.00289 \\
\hline hsa-mir-505 & $5.28 \pm 0.87$ & $4.56 \pm 1.24$ & 1.65 & 0.000014 & 0.0000407 \\
\hline hsa-mir-337 & $3.64 \pm 1.37$ & $2.93 \pm 0.85$ & 1.64 & 0.0039896 & 0.00719 \\
\hline hsa-mir-186 & $8.03 \pm 0.58$ & $7.36 \pm 0.61$ & 1.59 & $<\mathrm{le}-07$ & $<\mathrm{le}-07$ \\
\hline hsa-mir-92b & $7.33 \pm 1.15$ & $6.67 \pm 1.28$ & 1.58 & 0.0020063 & 0.00398 \\
\hline hsa-mir-324 & $5.4 \pm 0.89$ & $4.75 \pm 1.13$ & 1.56 & 0.0001217 & 0.000301 \\
\hline hsa-mir-30e & $13.47 \pm 0.56$ & $|2.84 \pm 0.7|$ & 1.55 & $<\mathrm{le}-07$ & $<\mathrm{le}-07$ \\
\hline hsa-mir-2/2 & $2.8 \pm 1.25$ & $2.17 \pm 0.99$ & 1.55 & 0.0057283 & 0.0101 \\
\hline hsa-mir-130a & $6.04 \pm 1.02$ & $5.42 \pm 1.3$ & 1.54 & 0.001206 & 0.00257 \\
\hline hsa-mir-335 & $6.79 \pm 0.94$ & $6.17 \pm 2.02$ & 1.53 & 0.0014444 & 0.00301 \\
\hline hsa-mir-I55 & $8.6 \pm 1.06$ & $7.98 \pm 0.88$ & 1.53 & 0.0014863 & 0.00308 \\
\hline hsa-mir-659 & $0.39 \pm 1.06$ & $-0.22 \pm 1.08$ & 1.53 & 0.0023122 & 0.00455 \\
\hline hsa-mir-28 & $11.96 \pm 0.65$ & $11.36 \pm 0.53$ & 1.52 & 0.0000005 & 0.0000018 \\
\hline hsa-mir-|8|b-I & $9.39 \pm 0.89$ & $8.78 \pm 0.51$ & 1.52 & 0.0001596 & 0.000392 \\
\hline hsa-mir-I6-I & $8.92 \pm 0.64$ & $8.33 \pm 1.65$ & 1.51 & 0.0000233 & 0.0000648 \\
\hline hsa-mir-I48b & $7.63 \pm 0.78$ & $7.06 \pm 0.61$ & 1.48 & 0.000069 & 0.000178 \\
\hline hsa-mir-I28-I & $6.21 \pm 0.94$ & $5.65 \pm 0.63$ & 1.47 & 0.0009856 & 0.00213 \\
\hline hsa-mir-200c & $13.16 \pm 0.92$ & $|2.6| \pm 0.8 \mid$ & 1.46 & 0.0011298 & 0.00242 \\
\hline hsa-mir-10a & $14.35 \pm 0.97$ & $13.8 \pm 1.14$ & 1.46 & 0.0025228 & 0.00494 \\
\hline hsa-mir-589 & $6.52 \pm 0.85$ & $6.06 \pm 0.96$ & 1.37 & 0.0039521 & 0.00719 \\
\hline hsa-mir-106b & $9.17 \pm 0.89$ & $8.75 \pm 0.57$ & 1.34 & 0.0079862 & 0.0138 \\
\hline hsa-mir-103-I & $13.99 \pm 0.72$ & $13.58 \pm 0.58$ & 1.32 & 0.0020074 & 0.00398 \\
\hline hsa-mir-26b & $9.33 \pm 0.7$ & $8.94 \pm 0.89$ & 1.31 & 0.0032529 & 0.00614 \\
\hline hsa-mir-27b & $10.74 \pm 0.77$ & $10.36 \pm 0.63$ & 1.31 & 0.0055184 & 0.00977 \\
\hline hsa-mir-125a & $9.25 \pm 0.69$ & $9.68 \pm 1.3$ & 0.74 & 0.0015033 & 0.00309 \\
\hline hsa-let-7g & $9.28 \pm 0.68$ & $9.77 \pm 1.24$ & 0.72 & 0.0003311 & 0.000777 \\
\hline hsa-mir-320a & $8.29 \pm 0.88$ & $8.76 \pm 0.55$ & 0.72 & 0.0031799 & 0.00607 \\
\hline hsa-mir-30d & $13.34 \pm 0.96$ & $13.84 \pm 0.6$ & $0.7 \mathrm{I}$ & 0.003882 & 0.00711 \\
\hline hsa-mir-99a & $7.97 \pm 1.07$ & $8.5 \pm 1.18$ & 0.69 & 0.0070379 & 0.0122 \\
\hline hsa-let-7b & $13.79 \pm 0.72$ & $14.36 \pm 0.99$ & 0.67 & 0.0000312 & 0.0000838 \\
\hline hsa-mir-193a & $7.38 \pm 1.08$ & $7.97 \pm I . I$ & 0.67 & 0.0034317 & 0.00644 \\
\hline hsa-mir-30c-2 & $8.69 \pm 0.79$ & $9.3 \pm 0.41$ & 0.66 & 0.0000184 & 0.0000521 \\
\hline hsa-mir-340 & $4.53 \pm 1$ & $5.14 \pm 1.87$ & 0.65 & 0.0029427 & 0.00565 \\
\hline hsa-mir-| 468 & $1.69 \pm 1.34$ & $2.32 \pm 0.8 I$ & 0.65 & 0.0091377 & 0.0157 \\
\hline hsa-mir-I5b & $7.67 \pm 0.77$ & $8.31 \pm 0.72$ & 0.64 & 0.0000075 & 0.0000226 \\
\hline hsa-mir-652 & $5.26 \pm 1.01$ & $5.91 \pm 0.7$ & 0.64 & 0.0003747 & 0.000867 \\
\hline hsa-let-7f-I & $4.3 I \pm 0.86$ & $4.95 \pm 1.89$ & 0.64 & 0.0005407 & 0.00121 \\
\hline hsa-mir-145 & $9.98 \pm 1.01$ & $10.61 \pm 1.31$ & 0.64 & 0.0008178 & 0.00179 \\
\hline hsa-mir-5II-I & $3.26 \pm 1.31$ & $3.94 \pm 1.01$ & 0.62 & 0.0044798 & 0.00802 \\
\hline hsa-mir-223 & $7.75 \pm 1.25$ & $8.57 \pm 1.36$ & 0.57 & 0.0004184 & 0.000961 \\
\hline hsa-mir-204 & $1.38 \pm 1.72$ & $2.23 \pm 1.25$ & 0.55 & 0.006179 & 0.0108 \\
\hline hsa-mir-548b & $|| \pm.|.5|$ & $2.01 \pm 1.16$ & 0.53 & 0.0018735 & 0.00378 \\
\hline hsa-mir-2।8-2 & $5.34 \pm 1.14$ & $6.33 \pm 2.56$ & 0.51 & 0.0000504 & 0.000132 \\
\hline hsa-let-7d & $9.03 \pm 0.69$ & $10.03 \pm 1.5$ & 0.5 & $<\mathrm{le}-07$ & $<\mathrm{le}-07$ \\
\hline hsa-let-7f-2 & $12.98 \pm 1.03$ & $13.97 \pm 2.24$ & 0.5 & 0.000004 & 0.0000124 \\
\hline hsa-mir-36I4 & $1.96 \pm 1.33$ & $2.98 \pm 0.88$ & 0.49 & 0.0000237 & 0.0000653 \\
\hline hsa-mir-140 & $9.69 \pm 0.83$ & $10.76 \pm 0.4 \mid$ & 0.48 & $<\mathrm{le}-07$ & $<\mathrm{le}-07$ \\
\hline
\end{tabular}


Table SI (Continued)

\begin{tabular}{|c|c|c|c|c|c|}
\hline \multirow[t]{2}{*}{ MicroRNA } & \multicolumn{2}{|c|}{ Expression level ${ }^{\mathrm{a}}$} & \multirow{2}{*}{$\begin{array}{l}\text { Fold } \\
\text { change }\end{array}$} & \multirow[t]{2}{*}{$P$-value } & \multirow[t]{2}{*}{ FDR } \\
\hline & LUAD & Normal & & & \\
\hline hsa-let-7e & $10.21 \pm 1.06$ & I I. $27 \pm 0.92$ & 0.48 & $<\mathrm{le}-07$ & 0.000000391 \\
\hline hsa-mir-598 & $3.56 \pm 1.24$ & $4.61 \pm 2.09$ & 0.48 & 0.000016 & 0.0000457 \\
\hline hsa-mir-I976 & $3.6 I \pm I . I I$ & $4.69 \pm 1.34$ & 0.47 & 0.0000002 & 0.000000755 \\
\hline hsa-let-7a-2 & $13.83 \pm 0.75$ & $14.97 \pm 0.35$ & 0.46 & $<\mathrm{Ie}-07$ & $<\mathrm{le}-07$ \\
\hline hsa-mir-| 38-I & $0.67 \pm 1.66$ & $1.8 \pm 1.58$ & 0.46 & 0.0002348 & 0.000564 \\
\hline hsa-mir-3912 & $-0.22 \pm I .1$ & $0.94 \pm 0.85$ & 0.45 & 0.0000002 & 0.000000755 \\
\hline hsa-mir-2II0 & $1.24 \pm 1.15$ & $2.43 \pm 1.31$ & 0.44 & $<\mathrm{le}-07$ & $<\mathrm{le}-07$ \\
\hline hsa-let-7a-I & $|2.63 \pm 0.7|$ & $13.89 \pm 0.42$ & 0.42 & $<\mathrm{le}-07$ & $<\mathrm{le}-07$ \\
\hline hsa-let-7a-3 & $12.72 \pm 0.72$ & $13.98 \pm 0.45$ & 0.42 & $<\mathrm{le}-07$ & $<\mathrm{le}-07$ \\
\hline hsa-mir-195 & $5.07 \pm 1.05$ & $6.49 \pm 1.45$ & 0.37 & $<\mathrm{le}-07$ & $<\mathrm{le}-07$ \\
\hline hsa-mir-1 25b-2 & $3.2 \pm 1.31$ & $4.63 \pm 0.62$ & 0.37 & $<\mathrm{le}-07$ & $<\mathrm{le}-07$ \\
\hline hsa-mir-5l4-3 & $0.8 \pm 2.23$ & $2.23 \pm 1.76$ & 0.37 & 0.0008279 & 0.0018 \\
\hline hsa-mir-101-2 & $5 . \mid 4 \pm 1.11$ & $6.63 \pm 2.36$ & 0.36 & $<\mathrm{le}-07$ & $<\mathrm{le}-07$ \\
\hline hsa-mir-584 & $4.43 \pm 1.45$ & $5.97 \pm 0.72$ & 0.34 & $<\mathrm{le}-07$ & $<\mathrm{le}-07$ \\
\hline hsa-mir-378c & $1.96 \pm 1.25$ & $3.59 \pm 1.13$ & 0.32 & $<\mathrm{le}-07$ & $<\mathrm{le}-07$ \\
\hline hsa-mir-2।8-I & $0.08 \pm 1.27$ & $1.76 \pm 1.24$ & 0.31 & $<\mathrm{le}-07$ & $<\mathrm{le}-07$ \\
\hline hsa-mir-| 43 & $|5.86 \pm|$ & $17.64 \pm 1.82$ & 0.29 & $<\mathrm{le}-07$ & $<\mathrm{le}-07$ \\
\hline hsa-let-7c & $9.98 \pm 1.1$ & I I.74 .0 .68 & 0.29 & $<\mathrm{le}-07$ & $<\mathrm{le}-07$ \\
\hline hsa-mir-30a & $13.85 \pm 1.12$ & $15.7 \pm 1.09$ & 0.28 & $<\mathrm{le}-07$ & $<\mathrm{le}-07$ \\
\hline hsa-mir-378 & $7.65 \pm 1.09$ & $9.57 \pm 0.97$ & 0.26 & $<\mathrm{le}-07$ & $<\mathrm{le}-07$ \\
\hline hsa-mir-I39 & $5.28 \pm 1.18$ & $7.26 \pm 1.25$ & 0.25 & $<\mathrm{le}-07$ & $<\mathrm{le}-07$ \\
\hline hsa-mir-I-2 & $2.78 \pm 1.76$ & $4.93 \pm 2.4$ & 0.22 & $<\mathrm{le}-07$ & $<\mathrm{le}-07$ \\
\hline hsa-mir-190 & $1.2 \pm 1.18$ & $3.43 \pm 1.29$ & 0.21 & $<\mathrm{le}-07$ & $<\mathrm{Ie}-07$ \\
\hline hsa-mir-45I & $8.35 \pm 1.6$ & $10.61 \pm 2.22$ & 0.21 & $<\mathrm{le}-07$ & $<\mathrm{le}-07$ \\
\hline hsa-mir-144 & $6.11 \pm 1.6$ & $8.46 \pm 3.27$ & 0.2 & $<\mathrm{Ie}-07$ & $<\mathrm{le}-07$ \\
\hline hsa-mir-I33a-I & 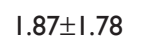 & $4.36 \pm I . I I$ & 0.18 & $<\mathrm{le}-07$ & $<\mathrm{le}-07$ \\
\hline hsa-mir-I 247 & $3.18 \pm 1.98$ & $5.76 \pm 1.18$ & 0.17 & $<\mathrm{le}-07$ & $<\mathrm{le}-07$ \\
\hline hsa-mir-184 & $1.07 \pm 2.11$ & $4.66 \pm 1.78$ & 0.083 & $<\mathrm{le}-07$ & $<\mathrm{le}-07$ \\
\hline hsa-mir-486 & $6.32 \pm 1.65$ & $10.11 \pm 1.48$ & 0.072 & $<\mathrm{le}-07$ & $<\mathrm{le}-07$ \\
\hline
\end{tabular}

Note: aLog2-transformed miRNA expression data, presented as mean \pm standard deviation.

Abbreviations: FDR, false discovery rate; LUAD, lung adenocarcinoma.

Table S2 MicroRNAs associated with the pathologic T of lung adenocarcinoma

\begin{tabular}{|c|c|c|c|c|c|}
\hline \multirow[t]{2}{*}{ MircoRNA } & \multicolumn{2}{|c|}{ Expression level $^{\mathrm{a}}$} & \multirow{2}{*}{$\begin{array}{l}\text { Fold } \\
\text { change }\end{array}$} & \multirow[t]{2}{*}{$P$-value } & \multirow[t]{2}{*}{ FDR } \\
\hline & $\mathbf{T} 3+\mathbf{T} 4$ & $\mathbf{T I}+\mathbf{T} 2$ & & & \\
\hline hsa-mir-186 & $7.8 I \pm 0.6 I$ & $8.07 \pm 0.57$ & 0.84 & 0.0022673 & 0.103 \\
\hline hsa-mir-103-2 & $2.93 \pm 0.79$ & $3.23 \pm 0.80$ & 0.81 & 0.0095228 & 0.216 \\
\hline hsa-mir-30e & $13.19 \pm 0.53$ & $|3.5| \pm 0.56$ & 0.8 & 0.0000848 & 0.0269 \\
\hline hsa-mir-26b & $9.05 \pm 0.60$ & $9.37 \pm 0.71$ & 0.8 & 0.0016008 & 0.0846 \\
\hline hsa-mir-454 & $2.60 \pm 0.90$ & $2.93 \pm 0.86$ & 0.79 & 0.0086132 & 0.21 \\
\hline hsa-mir-3653 & $2.12 \pm 1.10$ & $2.55 \pm 1.10$ & 0.74 & 0.0074348 & 0.206 \\
\hline hsa-mir-190 & $0.79 \pm 1.29$ & $1.24 \pm 1.14$ & 0.73 & 0.0077873 & 0.206 \\
\hline hsa-mir-345 & $3.49 \pm 1.27$ & $4.01 \pm 1.19$ & 0.7 & 0.0032425 & 0.128 \\
\hline hsa-mir-429 & $6.24 \pm 1.46$ & $6.81 \pm 1.15$ & 0.67 & 0.0009922 & 0.0629 \\
\hline hsa-mir-I42 & $\mid 0.62 \pm 1.31$ & $11.22 \pm 1.23$ & 0.66 & 0.0009389 & 0.0629 \\
\hline hsa-mir-3200 & $0.85 \pm 1.74$ & $1.49 \pm 1.53$ & 0.64 & $0.005425 I$ & 0.191 \\
\hline hsa-mir-30la & $3.04 \pm 1.22$ & $3.70 \pm 1.26$ & 0.63 & 0.0002553 & 0.0405 \\
\hline hsa-mir-|87 & $2.66 \pm 2.30$ & $3.78 \pm 2.28$ & 0.46 & 0.0007366 & 0.0629 \\
\hline
\end{tabular}

Note: aLog2-transformed miRNA expression data, presented as mean \pm standard deviation.

Abbreviation: FDR, false discovery rate. 
Table S3 MicroRNAs associated with the pathologic $\mathrm{N}$ of lung adenocarcinoma

\begin{tabular}{|c|c|c|c|c|c|}
\hline \multirow[t]{2}{*}{ MicroRNA } & \multicolumn{2}{|c|}{ Expression level ${ }^{a}$} & \multirow{2}{*}{$\begin{array}{l}\text { Fold } \\
\text { change }\end{array}$} & \multirow[t]{2}{*}{$P$-value } & \multirow[t]{2}{*}{ FDR } \\
\hline & $\mathbf{N} \mathbf{I}+\mathbf{N} 2+\mathbf{N} 3$ & No & & & \\
\hline hsa-mir-196b & $5.12 \pm 2.54$ & $4.45 \pm 2.44$ & 1.59 & 0.00993 & 0.236 \\
\hline hsa-mir-539 & $1.67 \pm 1.82$ & $1.20 \pm 1.60$ & 1.38 & 0.008377 & 0.236 \\
\hline hsa-mir-30c-2 & $8.54 \pm 0.80$ & $8.77 \pm 0.77$ & 0.85 & 0.004827 & 0.219 \\
\hline hsa-mir-660 & $5.06 \pm 1.17$ & $5.38 \pm 1.06$ & 0.8 & 0.00645 & 0.236 \\
\hline hsa-mir-30d & $13.11 \pm 0.94$ & $13.46 \pm 0.96$ & 0.78 & 0.00045 & 0.0476 \\
\hline hsa-mir-200a & $9.39 \pm 1.19$ & $9.75 \pm 0.98$ & 0.78 & 0.00121 & 0.0767 \\
\hline hsa-mir-200b & $8.97 \pm 1.22$ & $9.42 \pm 0.96$ & 0.73 & 0.0000619 & 0.00981 \\
\hline hsa-mir-204 & $1.04 \pm 1.67$ & $\mathrm{I} .5 \mathrm{I} \pm \mathrm{I} .74$ & 0.72 & 0.00969 & 0.236 \\
\hline hsa-mir-548b & $0.77 \pm 1.53$ & $1.26 \pm 1.48$ & 0.71 & 0.002651 & 0.14 \\
\hline hsa-mir-429 & $6.40 \pm 1.36$ & $6.91 \pm 1.10$ & 0.7 & 0.000061 & 0.00981 \\
\hline
\end{tabular}

Note: ${ }^{a}$ Log2-transformed miRNA expression data, presented as mean \pm standard deviation.

Abbreviation: FDR, false discovery rate.

Table S4 MicroRNAs associated with the pathologic Stage of lung adenocarcinoma

\begin{tabular}{|c|c|c|c|c|c|}
\hline \multirow[t]{2}{*}{ MicroRNA } & \multicolumn{2}{|c|}{ Expression level ${ }^{a}$} & \multirow{2}{*}{$\begin{array}{l}\text { Fold } \\
\text { change }\end{array}$} & \multirow[t]{2}{*}{$P$-value } & \multirow[t]{2}{*}{ FDR } \\
\hline & III + IV & I + II & & & \\
\hline hsa-mir-582 & $8.24 \pm 1.72$ & $7.73 \pm 1.56$ & 1.43 & 0.0064914 & 0.187 \\
\hline hsa-mir-30e & $|3.3| \pm 0.55$ & $13.52 \pm 0.56$ & 0.87 & 0.0017273 & 0.15 \\
\hline hsa-mir-29b-2 & $8.55 \pm 0.96$ & $8.88 \pm 0.95$ & 0.8 & 0.0037832 & 0.15 \\
\hline hsa-mir-29b-I & $8.40 \pm 0.99$ & $8.74 \pm 0.98$ & 0.79 & 0.003783 & 0.15 \\
\hline hsa-mir-200b & $9.00 \pm 1.26$ & $9.35 \pm 1.01$ & 0.78 & 0.0058496 & 0.185 \\
\hline hsa-mir-660 & $4.99 \pm 1.18$ & $5.38 \pm 1.06$ & 0.77 & 0.0031704 & 0.15 \\
\hline hsa-mir-200a & $9.28 \pm 1.22$ & $9.73 \pm 1.01$ & 0.73 & 0.0003346 & 0.106 \\
\hline hsa-mir-429 & $6.37 \pm 1.43$ & $6.84 \pm 1.13$ & 0.72 & 0.0009152 & 0.145 \\
\hline hsa-mir-548b & $0.7 I \pm I .55$ & $1.22 \pm 1.48$ & 0.7 & $0.005560 \mathrm{I}$ & 0.185 \\
\hline hsa-mir-3200 & $0.99 \pm 1.56$ & $1.55 \pm 1.53$ & 0.68 & 0.0025169 & 0.15 \\
\hline
\end{tabular}

Note: ${ }^{a}$ Log2-transformed miRNA expression data, presented as mean \pm standard deviation.

Abbreviation: FDR, false discovery rate.

\section{Publish your work in this journal}

OncoTargets and Therapy is an international, peer-reviewed, open access journal focusing on the pathological basis of all cancers, potential targets for therapy and treatment protocols employed to improve the management of cancer patients. The journal also focuses on the impact of management programs and new therapeutic agents and protocols on patient perspectives such as quality of life, adherence and satisfaction. The manuscript management system is completely online and includes a very quick and fair peer-review system, which is all easy to use. Visit http://www.dovepress.com/testimonials.php to read real quotes from published authors. 